\title{
Alcohol consumption leads to loss of healthy life, but the ADH1B*2 allele may still protect from NASH
}

\author{
Guillermo Mazzolini $^{1,2}$, Ali Canbay ${ }^{3}$ \\ ${ }^{1}$ Gene Therapy Laboratory, Instituto de Investigaciones en Medicina Traslacional (IIMT), Universidad Austral-CONICET, Buenos Aires, Argentina; \\ ${ }^{2}$ Liver Unit, Hospital Universitario Austral, Universidad Austral, Buenos Aires, Argentina; ${ }^{3}$ Department of Internal Medicine, University Hospital \\ Knappschaftskrankenhaus Bochum, Ruhr University Bochum, Bochum, Germany \\ Correspondence to: Ali Canbay, MD. Professor of Medicine, Director of the Department of Internal Medicine, University Hospital \\ Knappschaftskrankenhaus Bochum, Ruhr-University Bochum, In der Schornau 23-25, Bochum 44892, Germany. Email: ali.canbay@rub.de. \\ Comment on: Vilar-Gomez E, Sookoian S, Pirola CJ, et al. ADH1B*2 is associated with reduced severity of nonalcoholic fatty liver disease in adults, \\ independent of alcohol consumption. Gastroenterology 2020;159:929-43.
}

Submitted Jan 28, 2021. Accepted for publication May 19, 2021.

doi: $10.21037 /$ hbsn-21-49

View this article at: http://dx.doi.org/10.21037/hbsn-21-49

Nonalcoholic fatty liver disease (NAFLD) is now the most common cause of chronic liver disease worldwide, affecting almost a quarter of the world population $(1,2)$, and is a main cause of severe hepatic complications such as cirrhosis and liver cancer. In histological assessment NAFLD and alcoholic liver disease (ALD) are difficult to distinguish; NAFLD diagnosis thus requires a limit of alcohol intake. However, NAFLD and ALD very frequently coexist in the same patient, making it difficult to identify the exact cause of liver complications. Almost 5-6\% of all worldwide deaths ( $\sim 3$ million) are caused by the harmful use of alcohol (https://www.who.int/publications/i/item/9789241565639), therefore alcohol represents a major health problem at a global scale. There is an association between the amount of alcohol consumption and ALD, however, only $30 \%$ of chronic drinkers develop alcoholic hepatitis, and 10-20\% progress to advanced fibrosis or cirrhosis (3), underscoring the role of genetic factors involved in disease severity and progression. Any level of alcohol consumption, regardless of the amount, leads to loss of healthy life. The recommendation in clinical practice should be to avoid alcohol intake, particularly in the presence of any liver disease. Moderate alcohol consumption has been reported by many studies to be associated with less severe NAFLD, although some of these cross-sectional studies may be affected by selection bias (4-6). In contrast a longitudinal analysis of liver biopsies from patients with NAFLD showed that modest alcohol consumption was associated with lower
NASH resolution in comparison with nondrinkers (7). Recently, Chang et al. showed that the risk of liver steatosis in low and moderate alcohol consumers (MACs) was lower in comparison with nondrinkers (8); however, the proportion of hepatic steatosis plus fibrosis was higher in drinkers. The exact mechanism of the deleterious effect of alcohol consumption on NAFLD in obese patients is still unclear. When combined with free fatty acids the polyphenol resveratrol resulted in the stimulation of profibrogenic effects in hepatic stellate cells (key cells for induction and propagation of hepatic fibrosis) instead of any protective role (9). Therefore, it is important to shed light on the exact role of alcohol intake in addition to NAFLD to solve the currently rather conflicting data on this issue. The work published by Vilar-Gomez et al. in Gastroenterology (10) clarifies some knowledge gaps about alcohol metabolism and consumption, and the severity of NAFLD by studying the role of alcohol dehydrogenase $(\mathrm{ADH})-1 \mathrm{~B}$, in particular $A D H 1 B^{*} 2$, in this context. The authors started from a finding generated in a previous work by Sookoian et al., where they observed that patients with low alcohol intake and the $A D H 1 B^{*} 2$ allele showed a less severe NAFLD by histology compared to other patients studied (11). Vilar-Gomez et al. studied 1,697 patients enrolled into various studies conducted by the Nonalcoholic Steatohepatitis Clinical Research Network (NASH CRN) over a period of 10 years [2009-2019]; the NASH CRN Pathology committee reviewed all liver 
biopsies, and comprehensive alcohol consumption was obtained by AUDIT and LDH questionnaire, binge and heavy drinkers were excluded (170 patients). Remarkably, the frequency of $A D H 1 B * 2$ carriage varied across race being high in Asians/Pacific Islanders/Hawaiians (86\%) and low in non-Hispanic whites (8\%), Hispanics (14\%), and Blacks (4\%), but the study was focused on the 1,153 non-Hispanic whites, which were mainly female, obese, and hypertensive. Among the 1,153 patients, $30 \%$ had advanced fibrosis, and $60 \%$ had definite NASH. The cohort included 720 nondrinkers and 433 moderate drinkers, but no heavy alcohol consumers. $A D H 1 B^{*} 2$ carriers were more likely to be male, and moderate alcohol consumption was similar between $A D H 1 B^{*} 1$ and $A D H 1 B^{*} 2$ carriers.

$\mathrm{ADH}$ and aldehyde dehydrogenase (ALDH) are the primary enzymes involved in hepatic alcohol metabolism and there are variants that encode enzymes with different activity, vary in ethnic distribution, influencing the level of alcohol oxidation to the less toxic acetaldehyde, the level of alcohol consumption, and the risk of alcoholism or alcohol dependence (12). For example, the presence of the $A D H 1 B^{*} 2$ and $A D H 1 B^{*} 3$ alleles would be associated with a higher oxidative capacity leading to faster ethanol oxidation. Therefore, the presence of $A D H 1 B^{*} 2$ allele has shown a protective effect against alcohol dependence in Asian patients $(13,14)$. Vilar-Gomez et al. showed that, in comparison with non-drinkers, the prevalence of definite NASH (defined as NAFLD activity score $\geq 4$ ), and advanced fibrosis was lower in patients with MAC; moreover, they identified an inverse dose-dependent relationship between the amount of alcohol intake and risk of definite NASH up to a daily dose of $28 \mathrm{~g}$ alcohol. Although this was observed for both the $A D H 1 B^{*}$ and the $A D H 1 B^{*} 2$ allele, the NASH risk was significantly lower in patients carrying the $A D H 1 B * 2$ allele.

In summary, this study demonstrated a protective effect for patients carrying the $A D H 1 B^{*} 2$ allele on the risk of severe histological damage of NAFLD, including fibrosis. Patients with MAC have lower risk of definite NASH in a dose-dependent manner. The $A D H 1 B$ polymorphism $A D H B 1^{*} 2$ not only exerts a protective effect on NAFLD patients with MAC but also may reduce the calories gained by alcohol metabolism as patients had lower BMI in other studies (15). It seems that the beneficial effect of a highly metabolic ADH activity is diminished in patients with higher BMI with a limit of $37 \mathrm{~kg} / \mathrm{m}^{2}$ in the study of VilarGomez et al. Further studies are needed to clarify the cause for this BMI-dependent effect on NASH risk of this allele.
The limitations of the study as disclosed by the authors included possible misclassification of alcohol consumption by self-assessment, the lower number of Asians/Pacific Islanders/Hawaiians individuals included in the trial, lack of information on socio-economic patient status, physical activity and other possible confounding factors. Despite these limitations the present work demonstrates an unequivocal protective effect against NASH and NASH fibrosis by the $A D H B 1^{*} 2$ allele, which is independent of MAC but offset by very high BMI in a Caucasian population. This study opens the door for further studies in specific settings to unravel the exact mechanisms of the protection conferred by the $A D H B 1 * 2$ allele.

\section{Acknowledgments}

Funding: None.

\section{Footnote}

Provenance and Peer Review: This article was commissioned by the editorial office, Hepatobiliary Surgery and Nutrition. The article did not undergo external peer review.

Conflicts of Interest: Both authors have completed the ICMJE uniform disclosure form (available at https://hbsn. amegroups.com/article/view/10.21037/hbsn-21-49/coif). The authors have no conflicts of interest to declare.

Ethical Statement: The authors are accountable for all aspects of the work in ensuring that questions related to the accuracy or integrity of any part of the work are appropriately investigated and resolved.

Open Access Statement: This is an Open Access article distributed in accordance with the Creative Commons Attribution-NonCommercial-NoDerivs 4.0 International License (CC BY-NC-ND 4.0), which permits the noncommercial replication and distribution of the article with the strict proviso that no changes or edits are made and the original work is properly cited (including links to both the formal publication through the relevant DOI and the license). See: https://creativecommons.org/licenses/by-nc-nd/4.0/.

\section{References}

1. Younossi ZM, Koenig AB, Abdelatif D, et al. Global epidemiology of nonalcoholic fatty liver disease-Meta- 
analytic assessment of prevalence, incidence, and outcomes. Hepatology 2016;64:73-84.

2. Mazzolini G, Sowa JP, Atorrasagasti C, et al. Significance of simple steatosis: an update on the clinical and molecular evidence. Cells 2020;9:2458.

3. Teli MR, Day CP, Burt AD, et al. Determinants of progression to cirrhosis or fibrosis in pure alcoholic fatty liver. Lancet 1995;346:987-90.

4. Sookoian S, Castaño GO, Pirola CJ. Modest alcohol consumption decreases the risk of non-alcoholic fatty liver disease: a meta-analysis of 43175 individuals. Gut 2014;63:530-2.

5. Mitchell T, Jeffrey GP, de Boer B, et al. Type and pattern of alcohol consumption is associated with liver fibrosis in patients with non-alcoholic fatty liver disease. Am J Gastroenterol 2018;113:1484-93.

6. Yamada K, Mizukoshi E, Seike T, et al. Light alcohol consumption has the potential to suppress hepatocellular injury and liver fibrosis in non-alcoholic fatty liver disease. PLoS One 2018;13:e0191026.

7. Ajmera V, Belt P, Wilson LA, et al. Among patients with nonalcoholic fatty liver disease, modest alcohol use is associated with less improvement in histologic steatosis and steatohepatitis. Clin Gastroenterol Hepatol 2018;16:151120.e5.

8. Chang Y, Ryu S, Kim Y, et al. Low levels of alcohol consumption, obesity, and development of fatty liver with and without evidence of advanced fibrosis. Hepatology

Cite this article as: Mazzolini G, Canbay A. Alcohol consumption leads to loss of healthy life, but the $A D H 1 B^{*} 2$ allele may still protect from NASH. HepatoBiliary Surg Nutr 2021;10(3):391-393. doi: 10.21037/hbsn-21-49
2020;71:861-73.

9. Bechmann LP, Zahn D, Gieseler RK, et al. Resveratrol amplifies profibrogenic effects of free fatty acids on human hepatic stellate cells. Hepatol Res 2009;39:601-8.

10. Vilar-Gomez E, Sookoian S, Pirola CJ, et al. ADH1B*2 is associated with reduced severity of nonalcoholic fatty liver disease in adults, independent of alcohol consumption. Gastroenterology 2020;159:929-43.

11. Sookoian S, Flichman D, Castaño GO, et al. Mendelian randomisation suggests no beneficial effect of moderate alcohol consumption on the severity of nonalcoholic fatty liver disease. Aliment Pharmacol Ther 2016;44:1224-34.

12. Agarwal DP. Genetic polymorphisms of alcohol metabolizing enzymes. Pathol Biol (Paris) 2001;49:703-9.

13. Chen CC, Lu RB, Chen YC, et al. Interaction between the functional polymorphisms of the alcohol-metabolism genes in protection against alcoholism. Am J Hum Genet 1999;65:795-807.

14. Whitfield JB. Alcohol dehydrogenase and alcohol dependence: variation in genotype-associated risk between populations. Am J Hum Genet 2002;71:1247-50; author reply $1250-1$.

15. Yokoyama A, Yokoyama T, Matsui T, et al. Alcohol dehydrogenase-1B genotype (rs1229984) is a strong determinant of the relationship between body weight and alcohol intake in Japanese alcoholic men. Alcohol Clin Exp Res 2013;37:1123-32. 